\title{
IX. Therapeutics
}

Our increasing knowledge of the mechanisms of cell interactions opens the medical field to new treatments which place nanotechnology at the forefront of personalized medicine. Nanotechnology supports the development of more efficient targeted delivery of drugs and offers the possibility of decreasing the drug quantities used while obtaining the same or better results. Sometimes more elaborate therapeutic actions such as surgery and radiation exposure are needed. Nanotechnology can support minimally invasive surgical procedures and can also provide miniaturized implantable devices for long term monitoring and treatment of a patient.

\section{Drug delivery}

Medication constitutes the major portion of medical treatment. Modern pharmaceutics is evolving toward targeted drug delivery, a smart release of drug at the right place, at the right time, and in the right concentration. This can decrease the amount of drug delivered to a patient and provide a greater effectiveness of the treatment. A key point in targeted drug delivery is to minimize drug degradation and loss before it reaches the disease site. In current conventional methods of drug delivery, oral delivery for example, an excess amount of drug is given to the patient in order to get the right dose at the disease site. This excess can have negative side-effect on the patient. This is especially true in cancer chemotherapy where severe side-effects are often observed and need to be counteracted by other medications.

The perfect drug delivery system needs to have the following properties:

- It should protect the drug, allowing it to retain its biological and chemical effectiveness during its transit through the body to the target

- It should release the drug at the proper time and proper rate when the target is reached.

- It should minimize the toxicity and side-effects to healthy parts of the body as it performs its function.

One way to fulfill these conditions is to encapsulate the drug to protect it on its journey and to equip the vehicle carrying the drug with homing characteristics that carry it to the right place and a release mechanism allowing 


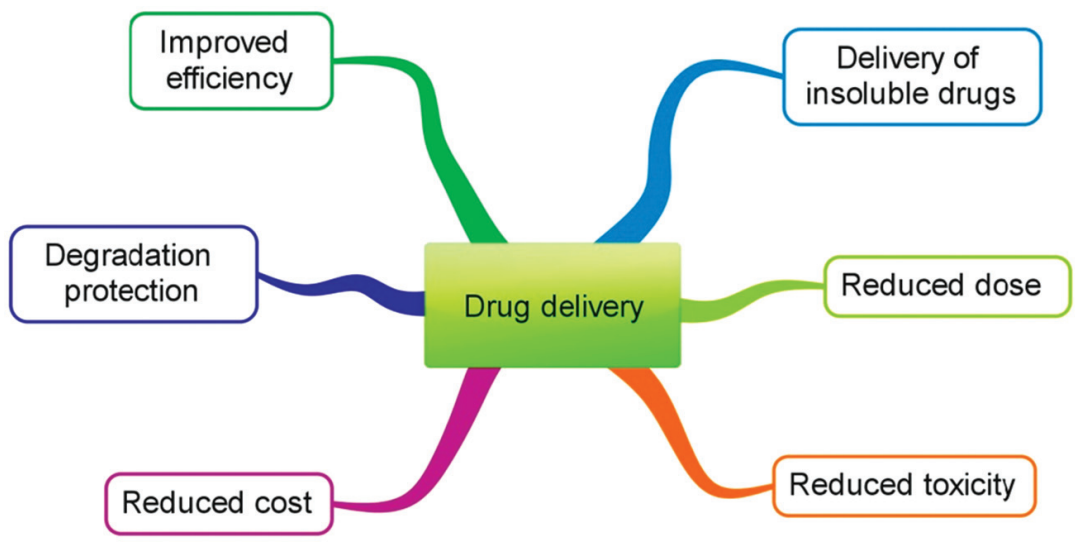

Figure 76. Advantages of nanotechnology in delivery of drugs.

optimal release of the drug on demand. Tailored nanoparticles have the ability to do that and represent a new way to control therapeutic treatments.

The expected benefits of introducing nanotechnology in drug delivery are summarized in figure 76 . Note that encapsulation offers the possibility of delivering insoluble drugs to the appropriate site, an important issue in some cancer chemotherapy treatments.

Nanotechnology also allows detailed control over the rate at which drugs are delivered. The delivery can be passive or active, continuous or pulsed. A passive delivery corresponds to the case where there is a self-degradation of the encapsulating material. In an active delivery the drug is released thanks to an external stimulus. Continuous delivery can be achieved by diffusion of the drug out of a biodegradable polymer, for example. Using an external stimulus such as light, $\mathrm{pH}$, temperature, electric field, etc. to trigger release of the drug allows a pulsed delivery.

\section{Delivery routes}

There are several routes for administration of medication. These are indicated in figure 77. The selection of the delivery method will ordinarily be based on efficiency and effectiveness for the particular disease to be treated.

The most common technique to deliver drugs is oral delivery. Oral medication is convenient in administration, well accepted by the patient and 


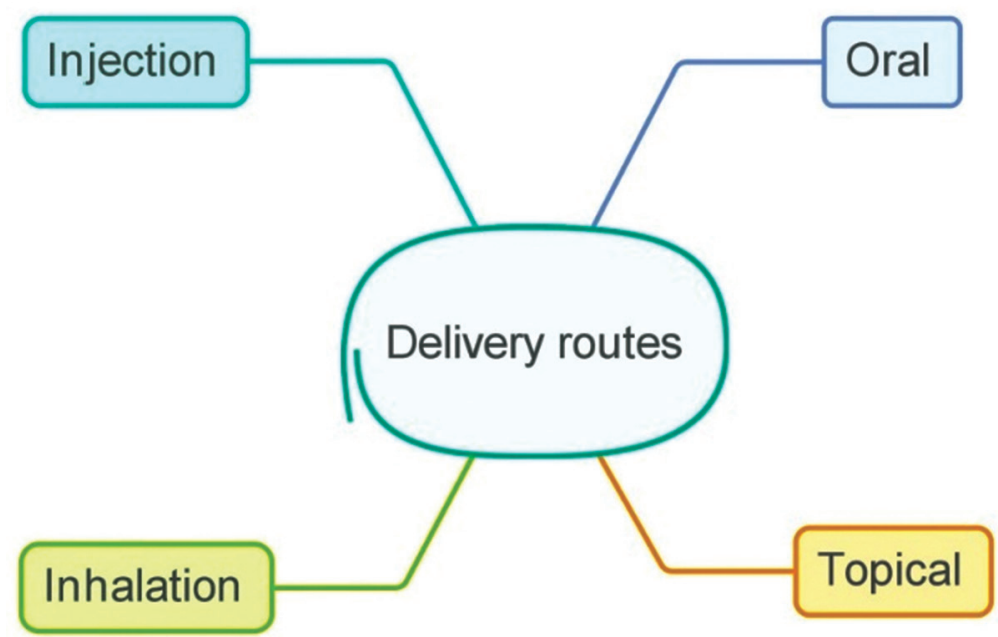

Figure 77. Different routes of administration of medication.

the manufacture of oral medicines is cost-effective. However, part of the drug may not reach the target leading to possible adverse side-effects.

Intravenous, intramuscular or subcutaneous injections are efficient ways to deliver drugs, but administration may be painful and require aseptic conditions for the patient.

Topical delivery consists in delivering the drug on or near the site to be cured, skin or mucous membranes for example.

Inhaled drugs are absorbed quickly and act locally. An inhaler device can be employed to deliver the right dose. Inhalation allows rapid drug delivery to the brain.

\section{Drug carriers}

Drug carriers, typically soluble entities or particulate matter of size less than one micrometer, offer the ability to deliver a drug to its intended target and to release it at that location. Many delivery strategies employ colloidal carriers. Colloidal systems encompass structures such as micelles, liposomes nanoparticles, etc. Micelles are formed with molecules having a hydrophilic (water attracting) head and a hydrophobic (water repelling) tail. Liposomes are vesicles formed with a lipid bilayer (figure 78). The hydrophilic head is directed toward the surrounding solvent (water) and the hydrophobic tail is directed inwards. A micelle can be formed with a variety 


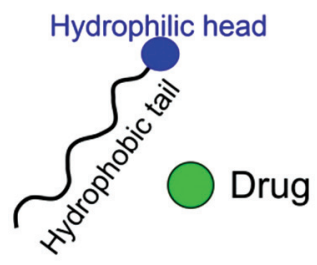

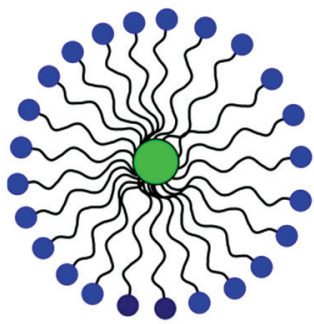

Micelle

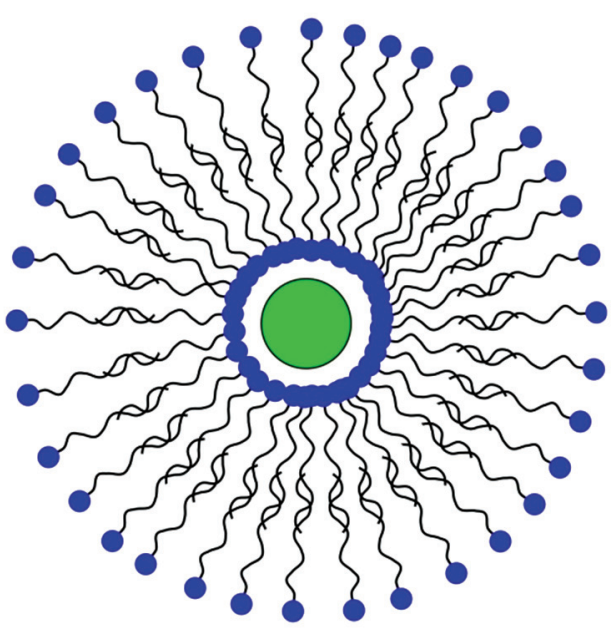

Liposome

Figure 78. Schematic representation of a micelle and a liposome.

of molecules such as fatty acids (soap), phospholipids, detergents, etc. In a micelle, the molecules form a hydrophilic shell and it is possible to put a molecule (drug) inside that shell. Especially interesting is the case in which the drug molecule itself is insoluble in water. The carrier makes it feasible to administer a larger concentration to the patient than is possible in the case of administering the free drug molecule. Furthermore, the shell protects the drug molecule until it is delivered to the right place.

The high selectivity of biological molecules, such as hormones or neurotransmitters, with respect to other molecules, is due to a "key-and-lock" mechanism (figure 79) in which a molecule can bind perfectly to another one. For example, a hormone (the key) binds to a receptor molecule. When this take place at the surface of a target cell, the properties of the cell are modified. Several drugs behave similarly to hormones and produce the same responses when they bind to the receptors. They are called agonists. Examples of agonists are morphine and nicotine. Other drugs (antagonists) behave in exactly the opposite way. When they bind to the receptor they do not produce the same response. Rather they inhibit responses. Examples of antagonists are caffeine, atropine and endocrine disruptors.

A molecular imprinted polymer has properties similar to those of active biological molecules. It behaves as a key to a receptor site molecule. This is schematically illustrated in figure 80 . Because of their similarities 


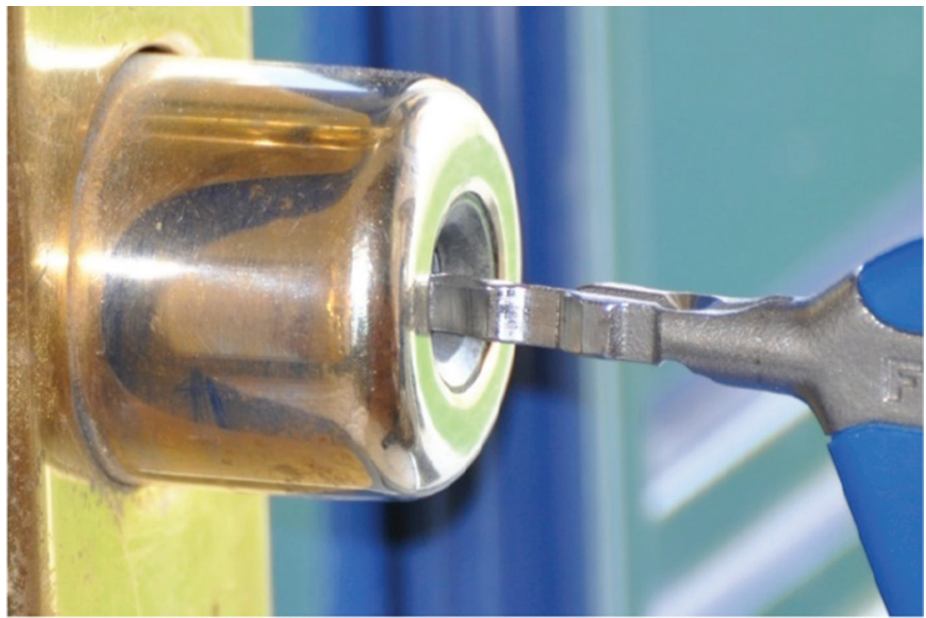

Figure 79. The key-and-lock scheme.
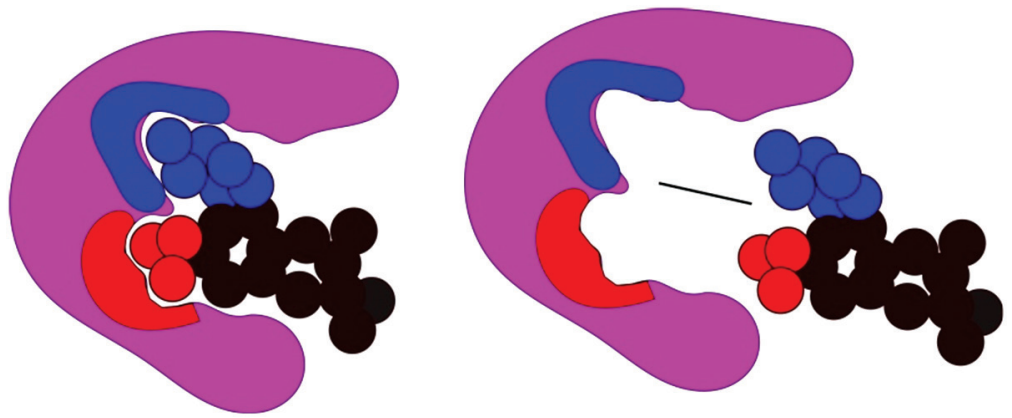

Figure 80 . Interaction of an imprinted polymer with a receptor.

to biological molecules, imprinted polymers have great potential in the field of drug delivery.

\section{Nanoparticles and drug delivery}

Nanoparticles provide several advantages for drug delivery compared to conventional methods. They are stable and can be designed to be highly specific. They can also carry a high dosage of drug while protecting it from outside interactions.

It is possible to graft specific molecules at the nanoparticle surface. These molecules can have different functions. Some molecules can make 


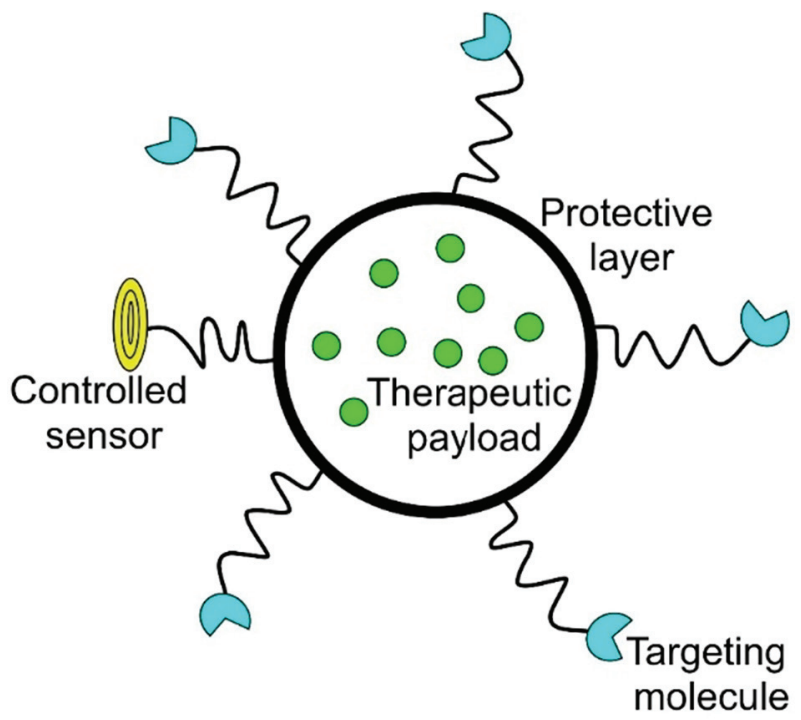

Figure 81. Illustration of a drug delivery nanoparticle. The nanoparticle, which can be a biodegradable polymer for example, encapsulates the drug (green circles) which constitutes the therapeutic payload. The nanoparticle can also contain an imaging contrast agent. The surface of the nanoparticle plays also the role of shield to make the nanoparticle invisible to the immune system. Targeting molecules grafted to the nanoparticle surface guide it and attach it to the cells of interest.

the nanoparticle invisible to the immune system so that they can travel through to the target without being attacked by the immune system. A typical illustration of this concept is shown in figure 81. Since the drug is protected until it reaches the target region, the side effects of the drug are minimized. Other molecules, with the ability to target specific cells, cancer cells, for example, can be attached. After reaching the target, the drug can be released using either passive or active techniques (see above). A schematic illustration of a functionalized nanoparticle is shown in figure 81 .

There are several families of nanoparticles that can be used to improve therapeutic treatments. This is a fast developing area and here we quote a few examples which illustrate the possibilities.

Drugs are often insoluble into water. Higher quantities than necessary are administrated to the patient to be sure that the right dose reaches the target region. This can lead to side effects because part of the drug is lost during the journey in the body of the patient. Encapsulating the drug into nanoparticles soluble in water can overcome this drawback. They are several such nanoparticle based techniques. A colloidal dispersion of nanoparticles is used for intravenous delivery of insoluble molecules. This allows more efficient delivery of the drug payload. 
Colloidal gold has been known since ancient times. Gold nanoparticles are easily manufactured and functionalized and have good biocompatibility. They are used in the treatment of rheumatoid arthritis and sclerosis. Small size gold nanoparticles, typically with diameters smaller than 5onm, have the ability to cross the brain-blood barrier. This is an interesting property which allows transfer of drugs from the blood to the brain.

Protein nanoparticles are often used for drug delivery. Albumin nanoparticles are used to deliver paclitaxel, a cancer drug, isolated from the bark of the Pacific yew tree. Paclitaxel is a highly toxic hydrophobic product. It is used in chemotherapy to treat lung, breast and ovarian cancers. Paclitaxel has many possible side effects such as a decrease in white blood cells, red cells and platelets; hair loss, inflammation of the mucous membranes, damage of the nerves, water retention, skin reactions, nail alteration...The cancer drug Abraxane is a solvent-free formulation of the paclitaxel. Binding Paclitaxel to albumin nanoparticles that will, in turn, bind to the tumor tissue reduces the toxic side effects and $50 \%$ more drug can be delivered at the right place.

Chitosan is a polysaccharide obtained from crustaceans such as shrimps. It has several applications but an interesting one is that it has the ability to clot blood. Nanoparticles of chitosan can be included in bandage and hemostatic agents. Chitosan has also antibacterial properties.

Another helpful application of nanoparticles is thermotherapy. By time-resolved infrared imaging it is possible to know the exact location of a tumor in the body by locating nanoparticles, attached to cancer cells. After this is done, it is possible to heat this specific region by laser irradiation and kill the cancer cells. Another advantage of time-resolved infrared imaging is that it can find metastasis cells located far from the initial tumor allowing the ability of an early treatment.

As a last example, consider carbon nanotubes. Either open or closed at one end (nanohorns). they can be used to encapsulate molecules, proteins and nucleotides. Their surfaces can also be functionalized. Carbon nanotubes have the ability to penetrate into cells without harming them, thanks to their very small size. Carbon nanotubes are particularly useful to deliver insoluble and toxic drugs because they are encapsulated and protected.

\section{Summary}

Drug delivery is an essential tool in health treatment. The goal is to deliver the right quantity at the right place and at the right time in order to 
maximize efficiency and minimize any side-effects. Nanoparticles have many advantages for drug delivery compared to conventional techniques. Drugs can be attached to nanoparticles that are eventually functionalized to go at the right place in the patient's body. Drug molecules can be also encapsulated within a functionalized nanoparticle that guides and releases them at the right place while protecting them during the travel in the body of the patient. With these new techniques, the trend towards dedicated treatments and personalized medicine is greatly accelerated. 